\title{
Comparison of Lyme Disease in the United States and Europe
}

\author{
Adriana R. Marques, Franc Strle, Gary P. Wormser
}

\begin{abstract}
Lyme disease, or Lyme borreliosis, is the most common tickborne disease in the United States and Europe. In both locations, Ixodes species ticks transmit the Borrelia burgdorferi sensu lato bacteria species responsible for causing the infection. The diversity of Borrelia species that cause human infection is greater in Europe; the 2 $B$. burgdorferi s.l. species collectively responsible for most infections in Europe, B. afzelii and B. garinii, are not found in the United States, where most infections are caused by $B$. burgdorferi sensu stricto. Strain differences seem to explain some of the variation in the clinical manifestations of Lyme disease, which are both minor and substantive, between the United States and Europe. Future studies should attempt to delineate the specific virulence factors of the different species of $B$. burgdorferi s.l. responsible for these variations in clinical features.
\end{abstract}

\begin{abstract}
T yme disease, or Lyme borreliosis, is the most common tickborne disease in both the United States and Europe; an estimated $\approx 476,000$ cases are diagnosed and treated per year in the United States and $>200,000$ cases per year in western Europe (1-3). The principal tick vector in the United States is Ixodes scapularis, followed by I. pacificus; in Europe, most cases are transmitted by I. ricinus, followed by I. persulcatus ticks (Table 1). The etiologic agent, Borrelia burgdorferi, was discovered in 1982 in the United States. Later, it became recognized that strains of B. burgdorferi in Europe were more heterogenous than strains in North America. B. burgdorferi sensu lato was then classified into 3 main genospecies. The originally discovered genospecies was named $B$. burgdorferi sensu stricto. The second genospecies was named Borrelia garinii sp. nov., and the third was named Borrelia afzelii sp. nov. Recently, the taxonomy of the family Borreliaceae (and the genus Borrelia) has been revised into 2
\end{abstract}

Author affiliations: National Institutes of Health, Bethesda, Maryland, USA (A.R. Marques); University Medical Centre Ljubljana, Ljubljana, Slovenia (F. Strle); New York Medical College, Valhalla, New York, USA (G.P. Wormser)

DOI: https://doi.org/10.3201/eid2708.204763 main genera, Borrelia and Borreliella (4). The spirochetes that cause relapsing fever retained the genus name Borrelia, and spirochetes that cause Lyme disease have been renamed Borreliella (hereafter referred to as Lyme borrelia). However, these changes have been challenged (5).

Most cases of Lyme disease in the United States occur in the mid-Atlantic, Northeast, and Upper Midwest regions. B. burgdorferi s.s., which also is found in Europe, causes most human infections in the United States $(1,2)$; the newly recognized species $B$. mayonii (which is not known to exist in Europe) is an infrequent cause of human illness in the Upper Midwest region of the United States (6). The incidence of Lyme disease in Europe is highest in the Scandinavian and Baltic states in northern Europe and in Austria, the Czech Republic, Germany, and Slovenia in central Europe. B. afzelii and B. garinii are the genospecies most frequently detected in I. ricinus and I. persulcatus ticks and cause most cases of Lyme disease in Europe $(1,2)$. Neither genospecies is found in the United States. Transmission of B. burgdorferi s.s. by I. scapularis or by $I$. pacificus ticks is very infrequent during the first 36 hours after tick attachment; in contrast, transmission of B. afzelii by I. ricinus ticks may occur within 24 hours (Table 1) (7).

\section{Erythema Migrans and Other Skin Manifestations}

After Lyme borrelia are deposited in the skin by the bite of an infected Ixodes tick, an infection is typically established at that site, which causes the characteristic skin lesion, erythema migrans (Figure $1)$. Erythema migrans is the most common clinical manifestation of Lyme disease in the United States and Europe, occurring in $\geq 80 \%$ of patients in both geographic areas (2). Overall, US patients with erythema migrans caused by B. burgdorferi s.s. are less likely than patients in Europe with erythema migrans caused by B. afzelii or B. garinii to remember a tick bite at the site of the lesion $(25 \%$ vs. $60 \%$ for $B$. afzelii or $64 \%$ for B. garinii) but more likely to have concomitant systemic symptoms $(69 \%$ vs. $38 \%$ or 
Table 1. Lyme disease in the United States and Europe

\begin{tabular}{|c|c|c|}
\hline Variable & United States & Europe \\
\hline Tick vector & Ixodes scapularis, I. pacificus & I. ricinus, I. persulcatus \\
\hline Lyme borrelia & $\begin{array}{c}\text { Mostly Borrelia burgdorferi sensu stricto; } B \text {. } \\
\text { mayonii may occur in the upper midwestern } \\
\text { United States }\end{array}$ & $\begin{array}{c}\text { Mostly } B \text {. afzelii and } B \text {. garinii, but several other } \\
\text { species cause human disease, including } B \text {. burgdorferi } \\
\text { s.s., B. bavariensis, B. spielmanii, and B. lusitaniae }\end{array}$ \\
\hline $\begin{array}{l}\text { Speed of tick transmission of } \\
\text { Lyme borrelia }\end{array}$ & Rarely before $36 \mathrm{~h}$ & I. ricinus ticks may transmit $B$. afzelii within $24 \mathrm{~h}$ \\
\hline Predominant patient sex & $\begin{array}{l}\text { Male patients account for } 56 \% \text { of reported } \\
\text { cases during } 2001-2018 \text {; no manifestation } \\
\text { is predominant among female patients }\end{array}$ & $\begin{array}{l}\text { Most cases of erythema migrans and acrodermatitis } \\
\text { chronica atrophicans occur in women; neuroborreliosis } \\
\text { and arthritis are predominant in men }\end{array}$ \\
\hline Coinfections & $\begin{array}{l}\text { Risk depends on the geographic area; the } \\
\text { most common co-infections are } \\
\text { anaplasmosis and babesiosis. }\end{array}$ & $\begin{array}{l}\text { Risk depends on the geographic area; the most } \\
\text { common co-infection is tick-borne encephalitis }\end{array}$ \\
\hline
\end{tabular}

$37 \%)$, multiple erythema migrans skin lesions (13\% vs. $5 \%$ for both B. afzelii and B. garinii), and regional lymphadenopathy $(29 \%$ vs. $8 \%$ or $3 \%)(8-10)$ (Table $2)$. Erythema migrans lesions in patients acquiring the infection in the United States have a shorter incubation period from tick bite to lesion development and are less likely to have central clearing at the time of diagnosis (8-10). The frequency of central clearing at least partially depends on the duration of the erythema migrans lesion before the diagnosis, and the duration is on average longer in Europe than in the United States (8-10). In Europe, the percentage of patients with multiple erythema migrans lesions is lower for adult patients than for children (8-11), whereas in the United States, multiple erythema migrans lesions occur with similar frequency in adults and children $(8,12-14)$. Patients infected with $B$. mayonii, found in the Upper Midwest region of the United States, can exhibit multiple and very small erythema migrans lesions (6).

In the United States, an entity referred to as southern tick-associated rash illness (STARI) is associated with a skin lesion very similar to erythema migrans (Figure 2).STARI, however, occurs after the bite of ticks of a different species, Amblyomma americanum, and is not caused by Lyme borrelia. The etiology of STARI has not been determined. A. americanum ticks are most frequently found in the southeastern and southcentral United States, but their range is spreading to geographic areas where I. scapularis tick bites are common (15). The potential for diagnostic confusion clearly exists in areas such as Long Island, New York, where both tick species coexist. STARI does not occur in Europe, presumably because A. americanum ticks are not found in that geographic area. Available, but limited, data suggest that STARI can be distinguished from erythema migrans on the basis of different serum metabolic profiles (16).

Two clinical manifestations of Lyme disease involving the skin occur exclusively in infections acquired in Europe: borrelial lymphocytoma and acrodermatitis chronica atrophicans (ACA) (Figure 3). Borrelial lymphocytoma appears as a small area of skin induration that slowly enlarges to a solitary bluish-red nodule or plaque with a diameter of up to a few centimeters and is predominantly located on the ear lobe in children and on the breast in adults. It usually develops at the site of a tick bite and is often accompanied with an erythema migrans lesion (17). ACA is a late cutaneous manifestation of Lyme disease located primarily on the extensor parts of the distal extremities. It starts with reddish-blue discoloration and swelling of the skin (an inflammatory phase), which slowly enlarges and, if untreated, is followed by atrophic changes several months to years later. For some patients, ACA was known to have been preceded by an earlier manifestation of Lyme disease, such as erythema
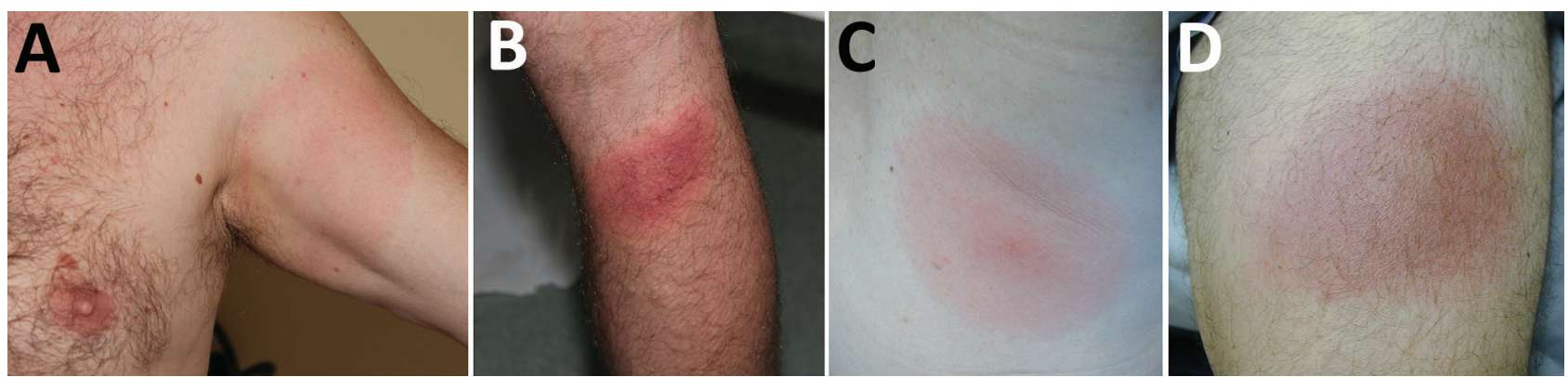

Figure 1. Erythema migrans skin lesions from patients in Europe (A, B) and the United States (C, D). 
Table 2. Characteristics of erythema migrans in the United States and Europe

\begin{tabular}{|c|c|c|c|}
\hline \multirow[b]{2}{*}{ Characteristic } & \multirow{2}{*}{$\begin{array}{c}\text { United States, \% cases } \\
\text { Borrelia burgdorferi sensu stricto* }\end{array}$} & \multicolumn{2}{|c|}{ Europe, $\%$ cases } \\
\hline & & B. afzelii† & B. gariniił \\
\hline Tick bite at skin site & 25 & 60 & 64 \\
\hline Central clearing & 35 & 69 & 62 \\
\hline Systemic symptoms & 69 & 38 & 37 \\
\hline Multiple erythema migrans lesions & 13 & 5 & 5 \\
\hline Regional lymphadenopathy & 29 & 8 & 3 \\
\hline
\end{tabular}

migrans (18). The apparent explanation for the absence of these manifestations in the United States is that these skin infections are principally caused by B. afzelii (Table 3).

\section{Neurologic Manifestations}

The typical presentation of early Lyme neuroborreliosis is cranial nerve palsy, particularly facial nerve palsy, as well as lymphocytic meningitis and painful radiculitis. In the United States, the most common manifestation of early Lyme neuroborreliosis is facial palsy. Most cases of early Lyme neuroborreliosis in Europe are caused by B. garinii and B. bavariensis; in adult patients, painful meningoradiculitis is most common $(19,20)$. In a study of 194 adult patients with Lyme neuroborreliosis in Denmark during 20152017 , radicular pain affected $70 \%$ of the patients and facial nerve palsy $43 \%$; intrathecal production of IgG or IgM against Lyme borrelia was found in $87 \%$ (21). Similar results were found in a retrospective series of 431 Lyme neuroborreliosis patients in Denmark, which included 126 children. Radicular pain (in 66\%) and facial nerve palsy (in $41 \%$ ) were the predominant symptoms; $84.5 \%$ of patients had evidence of intrathecal antibody production against Lyme borrelia (22). Although there are no comparable studies from the United States, it seems that adult US patients with early Lyme neuroborreliosis less frequently have severe radicular pain (23) (Table 3). Newer studies addressing Lyme neuroborreliosis in the United States would be a welcome addition for providing additional data on the frequency of particular symptoms and also on clarifying the frequency of intrathecal antibody production to B. burgdorferi s.s. at the time of symptom onset.

Late Lyme neuroborreliosis with encephalitis, myelitis, or encephalomyelitis has been reported in Europe but is very rare in the United States (24). On the other hand, 2 neurologic manifestations that have been reported to occur in the United States are now regarded as controversial. The first is Lyme encephalopathy, a poorly defined entity, which occurs in the absence of cerebrospinal fluid pleocytosis, intrathecal production of antiborrelial antibody, or direct microbiologic evidence of B. burgdorferi s.s. infection in the central nervous system. Symptoms include memory and concentration complaints. A now-recognized source of confusion with regard to this entity is that some patients with posttreatment Lyme disease syndrome in the United States report cognitive difficulties, and a subset of these patients have abnormal neurocognitive test results $(25,26)$. Adding to the controversy, however, is the question of what constitutes dysfunction on such testing and the clinical significance of the test results (27).

The second controversial neurologic manifestation in the United States is a chronic distal symmetric sensory neuropathy. In Europe, distal axonal neuropathy in the context of Lyme disease is exclusively associated with ACA. In patients with ACA, the neuropathy is predominantly sensory, most often in the involved skin areas (28). Case series in adult patients in the United States reported a similar neuropathy but without evidence of ACA $(29,30)$. The distribution of neurologic deficits, which is predominantly sensory, is distal and typically symmetric, but it can be asymmetric. The
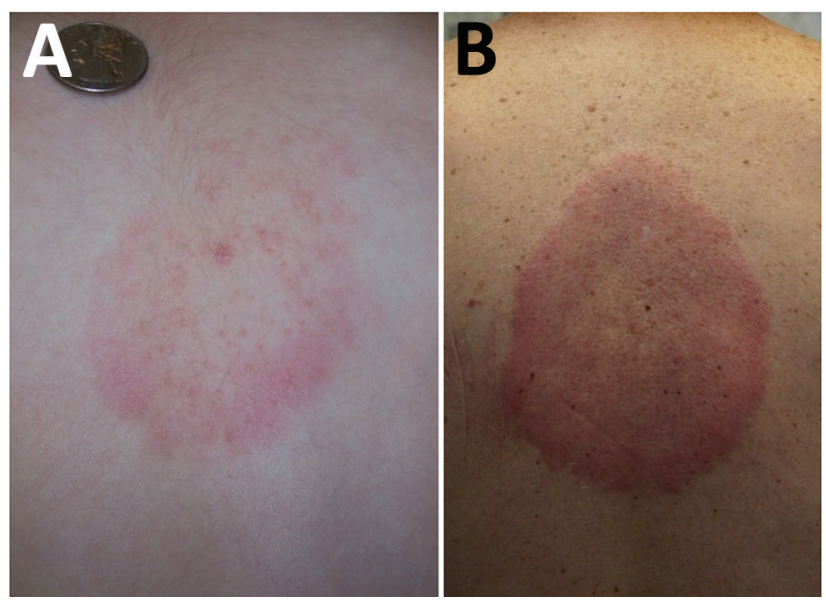

Figure 2. Southern tick-associated rash illness skin lesions. Adapted from Centers for Disease Control and Prevention, National Center for Emerging and Zoonotic Infectious Diseases, Division of Vector-Borne Diseases. 

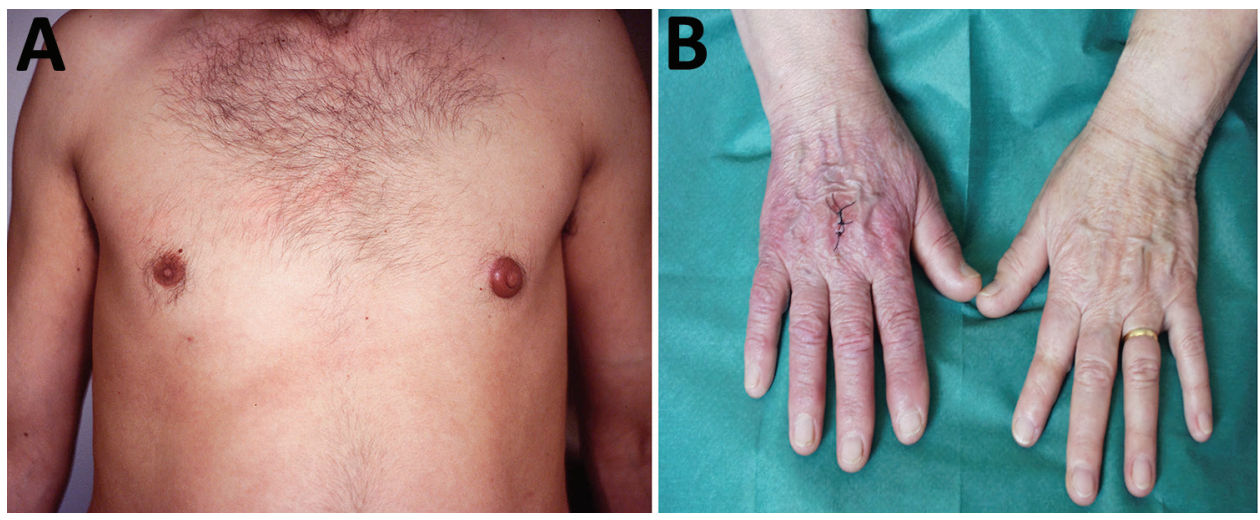

Figure 3. A) Borrelial lymphocytoma on nipple, showing local swelling and a remnant of erythema migrans on chest; at the time of diagnosis, the lesions had been noticed for 6 weeks. B) Acrodermatitis chronica atrophicans involving the right hand, showing redpurple discoloration, swelling, and skin atrophy; at the time of diagnosis, the lesions had been noticed for $\approx 2.5$ years. neuropathy is primarily axonal and thought to be a mononeuropathy multiplex, which can be confluent (24). Cerebrospinal fluid examination is usually unremarkable. Major concerns have been raised as to whether this entity has been appropriately validated as a manifestation of B. burgdorferi s.s. infection in the United States (31).

Overall, several factors have probably contributed to the belief that cognitive complaints or a chronic distal symmetric sensory peripheral neuropathy was attributable to Lyme disease in the United States (31). These factors include the use of diagnostic testing that is no longer considered valid, failure to appreciate that background seropositivity for antibodies to B. burgdorferi s.s. exists, and failure to include matched controls to determine if an association with cognitive complaints or peripheral neuropathy with a positive diagnostic assay for Lyme disease is higher than expected.

\section{Lyme Arthritis}

Lyme arthritis will develop in $\approx 60 \%$ of US patients with untreated erythema migrans over a 2-year period (32) and is said to comprise $28 \%$ of Lyme disease cases reported to the US Centers for Disease Control and Prevention that have data on symptoms available (33). Lyme arthritis seems to be less frequent in Europe $(34,35)$, and for untreated patients in Europe, the interval between onset of erythema migrans and development of Lyme arthritis may be shorter (1). Of note, B. burgdorferi s.s. was the most prevalent species of Lyme borrelia found in synovial fluid in a study of patients with Lyme arthritis in Europe (36). An acute manifestation of Lyme arthritis in children in the United States can mimic septic arthritis; this manifestation, however, does not seem to occur in children in Europe with Lyme arthritis (37).

With regard to demographics, Lyme disease in the United States is more common in male patients (56\% of the patients reported during 2001-2018 were male) (38). Indeed, no clinical manifestation has been associated with a female predominance in the United States, whereas in Europe, most cases of erythema migrans and ACA occur in women $(39,40)$. Many studies (but not all) demonstrated a male predominance for Lyme neuroborreliosis and Lyme arthritis $(22,35,36,41)$.

\section{Laboratory Diagnosis, Treatment, and Prophylaxis}

In the United States and Europe, most laboratory tests performed to diagnose Lyme disease are based on detecting serum antibodies to Lyme borrelia. Because Lyme disease in Europe is caused by a more diverse group of Lyme borrelia, criteria for test interpretation were more challenging to standardize than in the United States. In the United States, the Centers for Disease Controland Prevention has recommended the

\begin{tabular}{|c|c|c|}
\hline Manifestation & United States & Europe \\
\hline Radicular pain from Lyme neuroborreliosis & Less common in the United States* & More common in Europe \\
\hline Lyme arthritis & $\begin{array}{l}\text { More common in the United States in } \\
\text { untreated patients with erythema } \\
\text { migrans; may have septic arthritis-like } \\
\text { presentation in children }\end{array}$ & $\begin{array}{l}\text { Occurs in Europe; more commonly } \\
\text { associated with Borrelia burgdorferi sensu } \\
\text { stricto; septic arthritis-like manifestation in } \\
\text { children seems to be rare }\end{array}$ \\
\hline Acrodermatitis chronica atrophicans & No autochthonous US cases & Occurs in Europe (late manifestation) \\
\hline Borrelial lymphocytoma & No autochthonous US cases & Occurs in Europe \\
\hline Lyme encephalopathy & Controversial in the United States & Not recognized to occur \\
\hline Diffuse axonal peripheral neuropathy & Controversial in the United States & $\begin{array}{l}\text { Occurs but only in conjunction with } \\
\text { acrodermatitis chronica atrophicans }\end{array}$ \\
\hline
\end{tabular}

${ }^{\text {*} M o r e ~ s t u d i e s, ~ h o w e v e r, ~ a r e ~ n e e d e d . ~}$ 
standard 2-tier algorithm since 1995. This approach typically uses a sensitive enzyme immunoassay (EIA) as the initial step. A negative result requires no further testing. A positive or equivocal result is followed by supplemental testing using separate IgM and IgG immunoblots as the second-tier assay. The interpretation of immunoblot results uses standardized criteria (at least 2 of 3 signature bands for a positive IgM immunoblot and 5 of 10 signature bands for a positive IgG immunoblot). Results from the IgM immunoblot are only relevant when the duration of the illness is $<30$ days. Of note, testing performed in Europe is more likely to have positive results for patients who acquired Lyme disease in the United States than is testing performed in the United States to diagnose infection acquired in Europe (42). Recently, a 2-EIA approach has been approved as an alternative (or modified) 2-tier testing strategy (Figure 4). This new approach has higher sensitivity in early disease, similar specificity (43), greater ability for automation, and offers objective, quantitative values that leads to less variability in interpretation of the result. Also, the 2-EIA approach can be used in the United States and Europe. Moreover, it opens the door for a possible point-of-care test, a development that would be particularly helpful for patients with facial palsy, carditis, and pediatric patients with Lyme arthritis when septic arthritis is part of the differential diagnosis. A disadvantage is that the 2-EIA approach does not establish the extent of IgG seropositivity, which is essential knowledge for diagnosing late Lyme disease.

Recommendations for treating Lyme disease are generally very similar in guidelines for the United States and Europe. One difference is that phenoxymethylpenicillin (penicillin V) is recommended for treatment of erythema migrans and borrelial lymphocytoma by some of the guidelines in Europe but is not part of the treatment recommendations in the United States $(1,44,45)$. Another difference is the recommendation by some authorities in Europe to use intravenous ceftriaxone to treat erythema migrans, as well as other manifestations of Lyme disease, in pregnant women; whereas in the United States, antimicrobial drug treatment of Lyme disease for pregnant women is the same as that for nonpregnant patients, except that doxycycline is not recommended for pregnant women $(1,44,46)$. Postexposure antimicrobial prophylaxis with a single 200-mg dose of doxycycline has been shown to reduce the risk for Lyme disease after an I. scapularis tick bite and is recommended for consideration for tick bite prophylaxis in the United States (44). A recently published study conducted in Europe has also shown that a single 200-mg dose doxycycline successfully prevented Lyme disease after a tick bite (47). To what extent doxycycline will be used

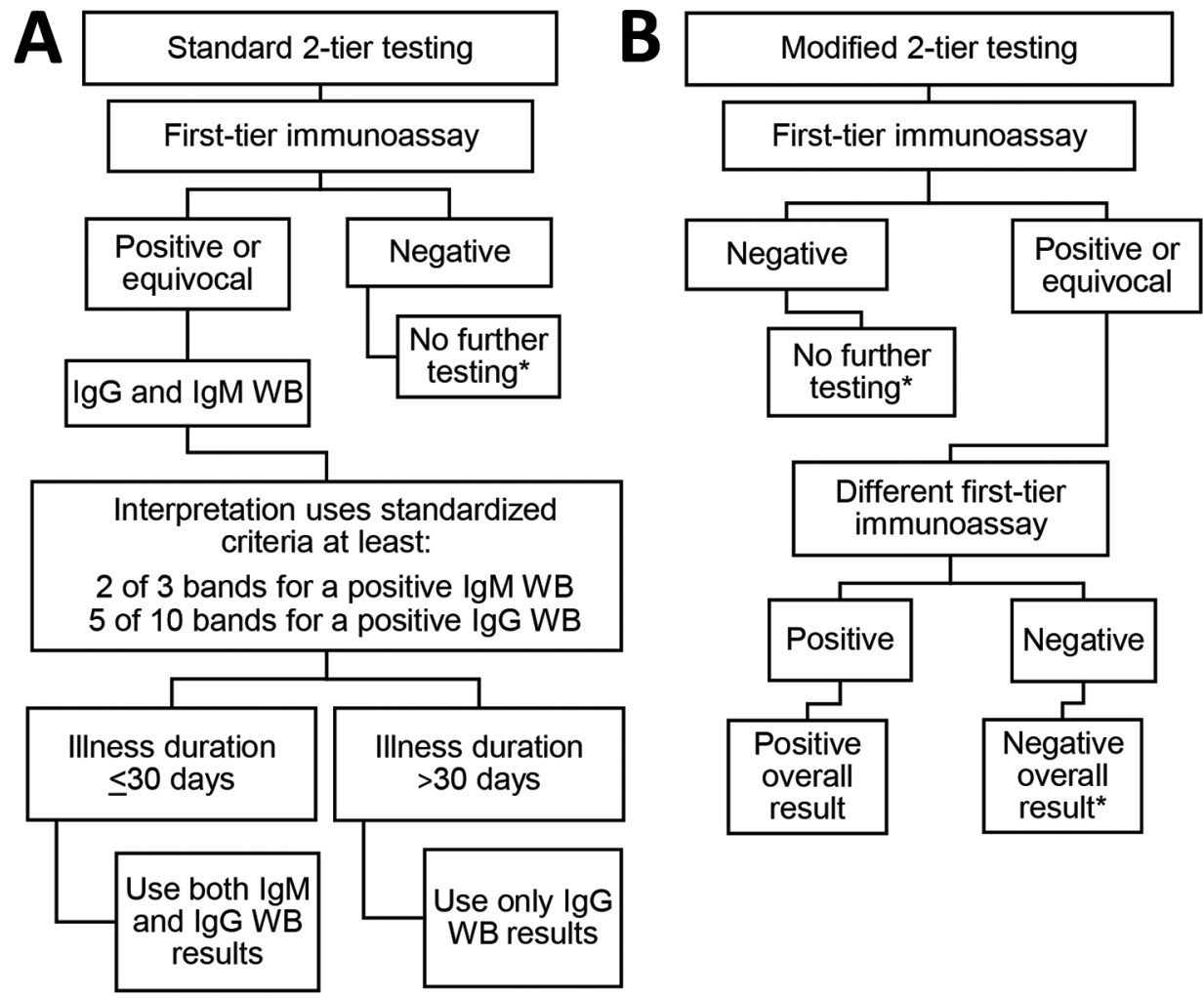

Figure 4. Standard 2-tier and modified 2-tier algorithms for serodiagnosis of Lyme disease. The US Centers for Disease Control and Prevention recommended a standard 2-tier algorithm $(A)$ and the modified 2-tier algorithm (B). ${ }^{*}$ For patients with signs or symptoms consistent with Lyme disease for $\leq 30$ days, the provider may treat and follow up with a convalescentphase serum sample. Patients with erythema migrans should receive treatment on the basis of the clinical diagnosis. WB, Western blot. 
in Europe after a tick bite is unknown; the standard of care has been observation (1).

\section{Lyme Borrelia Co-infections}

Ixodes ticks can carry multiple pathogens, and a single tick bite may result in transmission of $\geq 2$ infectious agents. Pathogens potentially transmitted by $I$. scapularis ticks to humans include B. burgdorferi s.s., B. mayonii, B. miyamotoi, Anaplasma phagocytophilum, Babesia microti, Ehrlichia muris eauclairensis, and the deer tick virus subtype of Powassan virus (48). The frequency of co-infections depends on the prevalence of the infectious agents in ticks, which will vary in different geographic areas. In the United States, $A$. phagocytophilum and B. microti are the most frequent co-infections in patients with Lyme disease (49). In the northeastern United States, $\approx 11 \%$ of patients infected with B. miyamotoi, a relapsing fever spirochete, are co-infected with B. burgdorferi s.s.; of note, B. miyamotoi infections per se can cause positive results on first-tier tests for Lyme disease, potentially leading to diagnostic confusion. Encephalitis caused by deer tick virus is relatively rare, but cases may be increasing. In Europe, in addition to Lyme borrelia, I. ricinus ticks can transmit tick-borne encephalitis virus, $A$. phagocytophilum, species of the bacterial genus Rickettsia, B. miyamotoi, and Babesia protozoans. Tick-borne encephalitis virus is well recognized as a cause of co-infection in patients with Lyme disease in Europe (50). More data are needed on the frequency of coinfections in both the United States and Europe.

\section{Conclusions}

Lyme disease is common in many areas of the United States and Europe and may have a variety of clinical manifestations. The duration of infection and the species of Lyme borrelia causing the infection can affect the clinical features of Lyme disease. In the United States, patients with erythema migrans more often have concomitant systemic symptoms than do patients in Europe. In Europe, Lyme arthritis is associated with $B$. burgdorferi s.s. and Lyme neuroborreliosis with B. garinii. Certain cutaneous manifestations of Lyme disease in Europe do not occur at all in the United States. It will be valuable to delineate the specific virulence factors of the different species of Lyme borrelia that contribute to these clinical differences.

Funding for this study was provided in part by the Division of Intramural Research, National Institute of Allergy and Infectious Diseases, National Institutes of Health.

A.R.M. has a patent US 8,926,989 issued and is an unpaid scientific advisor to the Global Lyme Alliance and to the
American Lyme Disease Foundation. F.S. served on the scientific advisory board for Roche on Lyme disease serologic diagnostics, on the scientific advisory board for Pfizer on Lyme disease vaccine, received research support from the Slovenian Research Agency (grant nos. P3-0296, J3-1744, and J3-8195), and is an unpaid member of the steering committee of the ESCMID Study Group on Lyme Borreliosis/ ESGBOR. G.P.W. received research grants from the Institute for Systems Biology and Pfizer, Inc. He owns equity in Abbott/ AbbVie, has been an expert witness in malpractice cases involving Lyme disease, and is an unpaid board member of the American Lyme Disease Foundation.

\section{About the Author}

Dr. Marques is chief of the Lyme Disease Studies Unit, Laboratory of Clinical Immunology and Microbiology, Division of Intramural Research, at the National Institute of Allergy and Infectious Diseases, National Institutes of Health, Bethesda, MD. Her primary research interest is clinical research on Lyme disease and other tickborne illnesses.

\section{References}

1. Stanek G, Strle F. Lyme borreliosis-from tick bite to diagnosis and treatment. FEMS Microbiol Rev. 2018;42:233-58. https://doi.org/10.1093/femsre/fux047

2. Steere AC, Strle F, Wormser GP, Hu LT, Branda JA, Hovius JW, et al. Lyme borreliosis. Nat Rev Dis Primers. 2016;2:16090. https:/ / doi.org/10.1038/nrdp.2016.90

3. Kugeler KJ, Schwartz AM, Delorey MJ, Mead PS, Hinckley AF. Estimating the frequency of lyme disease diagnoses, United States, 2010-2018. Emerg Infect Dis. 2021;27:616-9. https://doi.org/10.3201/eid2702.202731

4. Barbour AG, Gupta RS. The Family Borreliaceae (Spirochaetales), a diverse group in two genera of tick-borne spirochetes of mammals, birds, and reptiles. J Med Entomol. 2021:tjab055. https://doi.org/10.1093/jme/tjab055

5. Margos G, Castillo-Ramirez S, Cutler S, Dessau RB, Eikeland R, Estrada-Peña A, et al. Rejection of the name Borreliella and all proposed species comb. nov. placed therein. Int J Syst Evol Microbiol. 2020;70:3577-81. https://doi.org/10.1099/ijsem.0.004149

6. Pritt BS, Mead PS, Johnson DKH, Neitzel DF, Respicio-Kingry LB, Davis JP, et al. Identification of a novel pathogenic Borrelia species causing Lyme borreliosis with unusually high spirochaetaemia: a descriptive study. Lancet Infect Dis. 2016;16:556-64. https:/ / doi.org/10.1016/ S1473-3099(15)00464-8

7. Eisen L. Pathogen transmission in relation to duration of attachment by Ixodes scapularis ticks. Ticks Tick Borne Dis. 2018;9:535-42. https://doi.org/10.1016/j.ttbdis.2018.01.002

8. Strle F, Nadelman RB, Cimperman J, Nowakowski J, Picken RN, Schwartz I, et al. Comparison of cultureconfirmed erythema migrans caused by Borrelia burgdorferi sensu stricto in New York State and by Borrelia afzelii in Slovenia. Ann Intern Med. 1999;130:32-6. https:/ / doi.org/ 10.7326/0003-4819-130-1-199901050-00006

9. Strle F, Ružić-Sabljić E, Logar M, Maraspin V, Lotrič-Furlan $\mathrm{S}$, Cimperman J, et al. Comparison of erythema migrans caused by Borrelia burgdorferi and Borrelia garinii. Vector 
Borne Zoonotic Dis. 2011;11:1253-8. https:/ / doi.org/ 10.1089 /vbz.2010.0230

10. Logar M, Ruzić-Sabljić E, Maraspin V, Lotric-Furlan S, Cimperman J, Jurca T, et al. Comparison of erythema migrans caused by Borrelia afzelii and Borrelia garinii. Infection. 2004;32:15-9. https://doi.org/10.1007/s15010-004-3042-z

11. Arnez M, Pleterski-Rigler D, Luznik-Bufon T, Ruzic-Sabljić E, Strle F. Solitary and multiple erythema migrans in children: comparison of demographic, clinical and laboratory findings. Infection. 2003;31:404-9. https://doi.org/10.1007/ s15010-003-4007-3

12. Wormser GP, Ramanathan R, Nowakowski J, McKenna D, Holmgren D, Visintainer P, et al. Duration of antibiotic therapy for early Lyme disease. A randomized, double-blind, placebo-controlled trial. Ann Intern Med. 2003;138:697-704. https:/ / doi.org/10.7326/0003-4819-138-9-200305060-00005

13. Eppes SC, Childs JA. Comparative study of cefuroxime axetil versus amoxicillin in children with early Lyme disease. Pediatrics. 2002;109:1173-7. https:/ / doi.org/10.1542/ peds.109.6.1173

14. Gerber MA, Shapiro ED, Burke GS, Parcells VJ, Bell GL; Pediatric Lyme Disease Study Group. Lyme disease in children in southeastern Connecticut. N Engl J Med. 1996; 335:1270-4. https://doi.org/10.1056/NEJM199610243351703

15. Raghavan RK, Peterson AT, Cobos ME, Ganta R, Foley D. Current and future distribution of the lone star tick, Amblyomma americanum (L.) (Acari: Ixodidae) in North America. PLoS One. 2019;14:e0209082. https://doi.org/ 10.1371/journal.pone.0209082

16. Molins CR, Ashton LV, Wormser GP, Andre BG, Hess AM, Delorey MJ, et al. Metabolic differentiation of early Lyme disease from southern tick-associated rash illness (STARI). Sci Transl Med. 2017;9:eaal2717. https:/ / doi.org/10.1126/ scitranslmed.aal2717

17. Maraspin V, Nahtigal Klevišar M, Ružić-Sabljić E, Lusa L, Strle F. Borrelial lymphocytoma in adult patients. Clin Infect Dis. 2016;63:914-21. https://doi.org/10.1093/cid/ciw417

18. Ogrinc K, Maraspin V, Lusa L, Cerar Kišek T, Ružić-Sabljić E, Strle F. Acrodermatitis chronica atrophicans: clinical and microbiological characteristics of a cohort of 693 Slovenian patients. J Intern Med. 2021 Feb 7 [Epub ahead of print]. https://doi.org/10.1111/joim.13266

19. Coipan EC, Jahfari S, Fonville M, Oei GA, Spanjaard L, Takumi K, et al. Imbalanced presence of Borrelia burgdorferi s.l. multilocus sequence types in clinical manifestations of Lyme borreliosis. Infect Genet Evol. 2016;42:66-76. https:// doi.org/10.1016/j.meegid.2016.04.019

20. Ogrinc K, Lusa L, Lotrič-Furlan S, Bogovič P, Stupica D, Cerar T, et al. Course and outcome of early European Lyme neuroborreliosis (Bannwarth syndrome): clinical and laboratory findings. Clin Infect Dis. 2016;63:346-53. https://doi.org/10.1093/cid/ciw299

21. Nordberg CL, Bodilsen J, Knudtzen FC, Storgaard M, Brandt C, Wiese L, et al.; DASGIB study group. Lyme neuroborreliosis in adults: a nationwide prospective cohort study. Ticks Tick Borne Dis. 2020;11:101411. https:/ / doi.org/ 10.1016/j.ttbdis.2020.101411

22. Knudtzen FC, Andersen NS, Jensen TG, Skarphédinsson S. Characteristics and clinical outcome of Lyme neuroborreliosis in a high endemic area, 1995-2014: a retrospective cohort study in Denmark. Clin Infect Dis. 2017;65:1489-95. https://doi.org/10.1093/cid/cix568

23. Pachner AR, Steere AC. The triad of neurologic manifestations of Lyme disease: meningitis, cranial neuritis, and radiculoneuritis. Neurology. 1985;35:47-53. https:/ / doi.org/10.1212/WNL.35.1.47
24. Halperin JJ. Diagnosis and management of Lyme neuroborreliosis. Expert Rev Anti Infect Ther. 2018;16:5-11. https:/ / doi.org/10.1080/14787210.2018.1417836

25. Klempner MS, Hu LT, Evans J, Schmid CH, Johnson GM, Trevino RP, et al. Two controlled trials of antibiotic treatment in patients with persistent symptoms and a history of Lyme disease. N Engl J Med. 2001;345:85-92. https:// doi.org/10.1056/NEJM200107123450202

26. Krupp LB, Hyman LG, Grimson R, Coyle PK, Melville P, Ahnn S, et al. Study and treatment of post Lyme disease (STOP-LD): a randomized double masked clinical trial. Neurology. 2003;60:1923-30. https://doi.org/10.1212/ 01.WNL.0000071227.23769.9E

27. Binder LM, Iverson GL, Brooks BL. To err is human: "abnormal" neuropsychological scores and variability are common in healthy adults. Arch Clin Neuropsychol. 2009;24:31-46. https://doi.org/10.1093/arclin/acn001

28. Kindstrand E, Nilsson BY, Hovmark A, Pirskanen R, Asbrink E. Peripheral neuropathy in acrodermatitis chronica atrophicans - a late Borrelia manifestation. Acta Neurol Scand. 1997;95:338-45. https://doi.org/10.1111/j.1600-0404. 1997.tb00222.x

29. Logigian EL, Steere AC. Clinical and electrophysiologic findings in chronic neuropathy of Lyme disease. Neurology. 1992;42:303-11. https:/ / doi.org/10.1212/WNL.42.2.303

30. Halperin JJ, Little BW, Coyle PK, Dattwyler RJ. Lyme disease: cause of a treatable peripheral neuropathy. Neurology. 1987;37:1700-6. https://doi.org/10.1212/ WNL.37.11.1700

31. Wormser GP, Strle F, Shapiro ED, Dattwyler RJ, Auwaerter PG. A critical appraisal of the mild axonal peripheral neuropathy of late neurologic Lyme disease. Diagn Microbiol Infect Dis. 2017;87:163-7. https:// doi.org/10.1016/ j.diagmicrobio.2016.11.003

32. Steere AC, Malawista SE, Snydman DR, Shope RE, Andiman WA, Ross MR, et al. Lyme arthritis: an epidemic of oligoarticular arthritis in children and adults in three Connecticut communities. Arthritis Rheum. 1977;20:7-17. https://doi.org/10.1002/art.1780200102

33. Centers for Disease Control and Prevention. Lyme disease charts and figures: historical data [cited 2020 Nov 15]. https://www.cdc.gov/lyme/stats/graphs.html

34. Enkelmann J, Böhmer M, Fingerle V, Siffczyk C, Werber D, Littmann M, et al. Incidence of notified Lyme borreliosis in Germany, 2013-2017. Sci Rep. 2018;8:14976. https://doi.org/10.1038/s41598-018-33136-0

35. Strle F, Wormser GP, Mead P, Dhaduvai K, Longo MV, Adenikinju $\mathrm{O}$, et al. Gender disparity between cutaneous and non-cutaneous manifestations of Lyme borreliosis. PLoS One. 2013;8:e64110. https://doi.org/10.1371/ journal.pone.0064110

36. Grillon A, Scherlinger M, Boyer PH, De Martino S, Perdriger A, Blasquez A, et al. Characteristics and clinical outcomes after treatment of a national cohort of PCRpositive Lyme arthritis. Semin Arthritis Rheum. 2019;48:1105-12. https:// doi.org/10.1016/j.semarthrit.2018. 09.007

37. Huppertz HI, Karch H, Suschke HJ, Döring E, Ganser G, Thon A, et al.; The Pediatric Rheumatology Collaborative Group. Lyme arthritis in European children and adolescents. Arthritis Rheum. 1995;38:361-8. https://doi.org/10.1002/ art.1780380310

38. Centers for Disease Control and Prevention. Lyme disease charts and figures: most recent year [cited 2020 Nov 15]. https://www.cdc.gov/lyme/datasurveillance/ charts-figures-recent.html 
39. Sajanti E, Virtanen M, Helve O, Kuusi M, Lyytikäinen O, Hytönen J, et al. Lyme borreliosis in Finland, 1995-2014. Emerg Infect Dis. 2017;23:1282-8. https:/ / doi.org/10.3201/ eid2308.161273

40. Bennet L, Stjernberg L, Berglund J. Effect of gender on clinical and epidemiologic features of Lyme borreliosis. Vector Borne Zoonotic Dis. 2007;7:34-41. https:/ / doi.org/ 10.1089 /vbz.2006.0533

41. Rojko T, Bogovič P, Lotrič-Furlan S, Ogrinc K, Cerar-Kišek T, Glinšek Biškup U, et al. Borrelia burgdorferi sensu lato infection in patients with peripheral facial palsy. Ticks Tick Borne Dis. 2019;10:398-406. https://doi.org/10.1016/ j.ttbdis.2018.11.019

42. Wormser GP, Tang AT, Schimmoeller NR, Bittker S, Cooper D, Visintainer $\mathrm{P}$, et al. Utility of serodiagnostics designed for use in the United States for detection of Lyme borreliosis acquired in Europe and vice versa. Med Microbiol Immunol (Berl). 2014;203:65-71. https:/ / doi.org/10.1007/ s00430-013-0315-0

43. Marques AR. Revisiting the Lyme disease serodiagnostic algorithm: the momentum gathers. J Clin Microbiol. 2018;56:e00749-18. https://doi.org/10.1128/JCM.00749-18

44. Lantos PM, Rumbaugh J, Bockenstedt LK, Falck-Ytter YT, Aguero-Rosenfeld ME, Auwaerter PG, et al. Clinical Practice Guidelines by the Infectious Diseases Society of America (IDSA), American Academy of Neurology (AAN), and American College of Rheumatology (ACR): 2020 Guidelines for the Prevention, Diagnosis and Treatment of Lyme Disease. Clin Infect Dis. 2021;72:1-8.

45. Wormser GP, Strle F. Evaluation of the role of oral penicillin for treating Lyme disease patients with erythema migrans in the United States. Diagn Microbiol Infect Dis. 2020;97:115071. https://doi.org/10.1016/j.diagmicrobio.2020.115071

46. Maraspin V, Lusa L, Blejec T, Ružić-Sabljić E, Pohar Perme M, Strle F. Course and outcome of erythema migrans in pregnant women. J Clin Med. 2020;9:E2364. https://doi.org/ $10.3390 /$ jcm 9082364

47. Harms MG, Hofhuis A, Sprong H, Bennema SC, Ferreira JA, Fonville M, et al. A single dose of doxycycline after an Ixodes ricinus tick bite to prevent Lyme borreliosis: an open-label randomized controlled trial. J Infect. 2021;82:98-104. https://doi.org/10.1016/j.jinf.2020.06.032

48. Nelder MP, Russell CB, Sheehan NJ, Sander B, Moore S, Li Y, et al. Human pathogens associated with the blacklegged tick Ixodes scapularis: a systematic review. Parasit Vectors. 2016;9:265. https://doi.org/10.1186/ s13071-016-1529-y

49. Centers for Disease Control and Prevention. Nationally notifiable infectious diseases and conditions, United States: annual tables. Annual data for 2018. Tables 1, 2c, 2e [cited 2020 Nov 15]. https:/ / wonder.cdc.gov/nndss/ nndss_annual_tables_menu.asp?mmwr_year $=2018$

50. Velušček M, Blagus R, Cerar Kišek T, Ružić-Sabljić E, Avšič-Županc T. F Bajrović F, et al. Antibiotic use and long-term outcome in patients with tick-borne encephalitis and co-infection with Borrelia Burgdorferi sensu lato in central Europe. A retrospective cohort study J Clin Med. 2019;8:E1740. https://doi.org/10.3390/ jcm8101740

Address for correspondence: Adriana Marques. Laboratory of Clinical Immunology and Microbiology, National Institute of Allergy and Infectious Diseases, National Institutes of Health, 10/12C118 10 Center Dr, Bethesda, MD 20892, USA; email: amarques@niaid.nih.gov

\section{EID Podcast Livestock, Phages, MRSA, and People in Denmark}

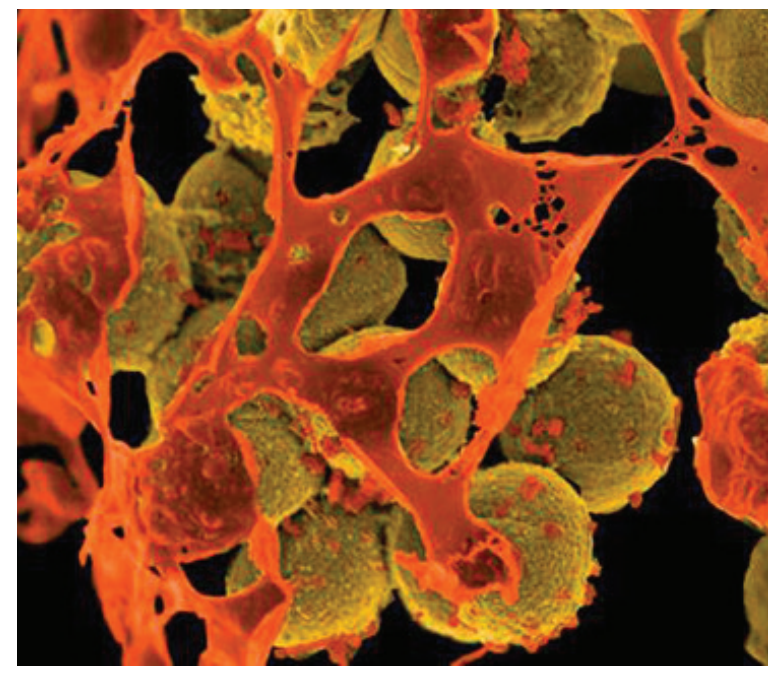

Methicillin-resistant Staphylococcus aureus, better known as MRSA, is often found on human skin. But MRSA can also cause dangerous infections that are resistant to common antimicrobial drugs. Epidemiologists carefully monitor any new mutations or transmission modes that might lead to the spread of this infection.

Approximately 15 years ago, MRSA emerged in livestock. From 2008 to 2018, the proportion of infected pigs in Denmark rocketed from 3.5\% to $90 \%$.

What happened, and what does this mean for human health?

In this EID podcast, Dr. Jesper Larsen, a senior researcher at the Statens Serum Institut, describes the spread of MRSA from livestock to humans.

Visit our website to listen: https: //go.usa.gov/x74Jh EMERGING INFECTIOUS DISEASES ${ }^{\circ}$ 